\title{
Italique
}

Poésie italienne de la Renaissance

I| 1998

Varia

\section{Storia e fortuna senesi di un sonetto di Galeazzo di Tarsia}

\section{Massimo Danzi}

\section{(2) OpenEdition}

\section{Journals}

\section{Edizione digitale}

URL: http://journals.openedition.org/italique/87

DOI: $10.4000 /$ italique. 87

ISSN: 1663-4438

\section{Editore}

Librairie Droz

\section{Edizione cartacea}

Data di pubblicazione: 1 giugno 1998

Paginazione: 61-78

ISBN: 2-600-00239-1

ISSN: 1423-3983

Notizia bibliografica digitale

Massimo Danzi, « Storia e fortuna senesi di un sonetto di Galeazzo di Tarsia », Italique [Online], I 1 1998, online dal 14 septembre 2009, consultato il 20 avril 2019. URL : http://journals.openedition.org/ italique/87 ; DOI : 10.4000/italique.87 
MASSIMO DANZI

STORIA E FORTUNA SENESI DI UN SONETTO DI GALEAZZO DI TARSIA 


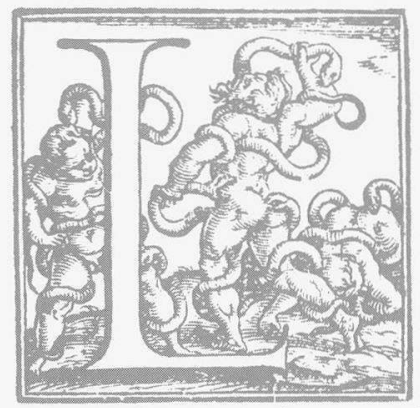

A circolazione della poesia del nobile, d'origine cosentina, Galeazzo di Tarsia ( $\mathrm{Na}$ poli 1520-1553), che nella geografia della lirica cinquecentesca si associa ai maggiori poeti napoletani quali Angelo Di Costanzo o Luigi Tansillo, e per l'altezza della sua meditazione amorosa richiama la gravitas del maggior lirico del secolo, Giovanni della Casa, ci è poco nota. Quel poco si trova, magistralmente indagato, nell'edizione che del poeta ha fornito, nel 1980, Cesare Bozzetti razionalizzando $i$ dati di una tradizione limitata ma complessa per il comportamento fortemente differenziato dei testimoni, dimostrati appartenere a fasi diverse di eleborazione del testo. ${ }^{1}$ Fra le novità dell'edizione, spicca quella di un commento che, funzionale alla nuova lezione stabilita, adduce testi prevalentemente contemporanei, con una scelta intesa a illuminare la poesia del Tarsia sullo sfondo dei testi e degli autori che le prime fortunate antologie poetiche avevano proprio in quegli anni rimesso in circolazione: dal Libro primo delle Rime diverse di molti eccellentissimi auttori del 1545 al Libro settimo delle Rime di diversi signori napoletani del 1556, per non ricordare che le due procurate a Venezia dal Giolito.

Studiando una nuova redazione di un sonetto di Galeazzo, anteriore ai materiali finora noti, e un più problematico frammento ad essa riportabile scoperti in un codice della Biblioteca degli Intronati di Siena, il presente contributo a quel magistero si ricollega, in un dialogo a distanza cui non è, negli anni, mai venuta meno la familiarità e l'incoraggiamento.

Il sonetto XVI di Galeazzo, che qui riporto insieme al breve apparato stabilito dall'edizione critica in due fasce di varianti, rispettivamente sostanziali sostanziali e formali, recita:

Come in limpido vetro o in onda pura,

Se il destr'occhio del ciel risplende in lui,

Mirar si può quel che ne' raggi sui

Debil vista mirar non s'assicura,

Così la mia, ch'altro veder non cura,

Perde, Donna real, mirando in vui

Che siete un nuovo sole oggi fra nui:

Ch'occhio non sano a gran splendor non dura. 


\section{MASSIMO DANZI}

Ma se mi volgo al cor che d'ogni parte

Riceve il folgorar del vago viso,

Non splende raggio in lui ch'ei non si mostri.

Dunque a che tormi il sol de gli occhi vostri

Se il veggio assai via men se in lui m'affiso

E lo scopro in me stesso a parte a parte ? ${ }^{2}$

4 mirar] a mirar $\mathrm{C}, \mathrm{V}-8$ splendor] fulgor $\mathrm{C}-10$ folgorar] balenar $\mathrm{C}$ - vago] vostro $\mathrm{C}, \mathrm{V}, \mathrm{NR}-11$ lui] voi $\mathrm{C}, \mathrm{V}, \mathrm{NR}$ - ch'ei] che $\mathrm{C}-13$ assai via men se in lui m'] meno assai qualor l' C

2 Se il] Se 'I C, V - destr'] destro C, V - 3 ne'] ne i NR - 4 assicura] assecura NR 7 siete] sete C, V, NR - nuovo] novo C, V - 12 de gli] degli NS - 13 Se il] Se'l V - se in] s'in V - 14 scopro] scuopro C, V

Tema del sonetto è la contemplazione dell'amata, che qui procede con un andamento definitorio che può richiamare $i$ sonetti VIII e XIV, e si esplica - riprendendo le parole del Bozzetti - con la tipica tendenza tarsiana "a far di fenomeni naturali simboli e metafore di situazioni psicologiche e affettive». Indubbia pare anche a me una componente uscolastica $e$ manierata", che fa del sonetto uno dei testi piu involuti e concettosi del canzoniere di Galeazzo e tale, ad ogni modo, da rendere la rappresentazione, fin dalla quartina d'inizio, non forse immediatamente perspicua. Così dovette apparire per lo meno ad un suo più antico editore "critico", il Bartelli, che provandosi nel 1888 a fissarne il testo non sapeva rinunciare a darne una sobria quanto sostanziale parafrasi, nei termini che riporto: "come occhio umano non può fissare il sole se non attraverso il vetro o l'acqua, così io, non potendo fissare voi, che siete un nuovo sole, vi miro riflessa nel mio cuore. Dunque è vano che vi nascondiate, perché vi scopro a parte a parte in me stesso».

Analizzando per primo il costituirsi del testo sulla base della varia lectio delle testimonianze a lui note, il Bozzetti ne illustrava l'elaborazione nei termini di "un iter correttorio che da C attraverso V e NR perviene all'assestamento finale di NS. Che è poi - aggiungeva - un iter dominato essenzialmente dalla ricerca di un lessico sempre piu petrarchesco e insieme da una progressiva attenuazione delle forme troppo colloquiali)., Da questo lucido quanto sicuro giudizio depositato nel commento al sonetto, si può partire per dar conto di un testimone che reca alla conoscenza di quell'elaborazione una nuova fase, preziosa perché arcaica al punto da coincidere con la più antica testimonianza manoscritta serbatasi di un testo di Galeazzo. 
Una versione lievemente diversa del sonetto ci è tramandata dal manoscritto I.XI.49 della Biblioteca Comunale degli Intronati di Siena, che lo contiene adespoto entro un gruppo di rime attribuite invece esplicitamente ad Antonio Barozzi, letterato d'origine genovese della prima metà del secolo e membro della Accademia degli Intronati di Siena con il nome di «Deserto». Il Barozzi non è, in verità, personaggio molto noto e le sue rime, fatta salva qualche stanza di cui si dirà in seguito, sono inedite. Del codice senese manca una descrizione, non essendo tale né la scheda dell'Ilari nel catalogo che dei manoscritti di quella biblioteca egli diede a metà dell'Ottocento, né quella moderna del sempre benemerito Iter italicum del Kristeller, che rispetto all'Ilari aggiunge il nome di qualche autore sulla base di un indice che una mano più tarda appose all'inizio del codice. ${ }^{6}$ Come nel caso del Tarsia, tuttavia, entrambi $i$ cataloghi tacciono sulla paternità di tutti quei testi che il codice tramanda adespoti. Si tratta di un miscellaneo di rime esclusivamente cinquecentesche databile in base a indizi interni fra 1550 e 1560 circa e che subito appare fortemente implicato con la realtà della città di Siena per la presenza, dichiarata (nel caso de "Il Deserto") o adespota (in altri numerosi casi), di esponenti dell'Accademia degli Intronati. Il manoscritto, che nelle prime sue 53 carte contiene solo testi volgari e poi, da c. $54 \mathrm{r}$ innanzi, invece esclusivamente testi latini, fu, assai probabilmente, confezionato proprio a Siena per successive aggiunte di materiali lì giunti da varie parti di Italia. Lo segnalano, a diverse riprese, le rubriche dei testi trascritti dove i diversi copisti registrano data e provenienza e anzi, per quanto attiene alle date, con una progressione cronologica che appare nel codice, con qualche eccezione, rispettata. ${ }^{8}$

La città di Siena costituisce d'altra parte una tappa importante ed estrema nella avventurosa biografia di Galeazzo. Andrà richiamato, infatti, come, condannato al confine nell'isola di Lipari nella seconda metà del 1547 per reati di violenze e soprusi commessi contro alcuni suoi feudatari, Galeazzo venisse graziato e tornasse, già nel luglio del 1552, nel suo feudo cosentino di Belmonte. Siamo, a tale altezza cronologica, immediatamente a ridosso della spedizione che contro Siena stava organizzando il Toledo. Liberato, Galeazzo dovette arruolarsi con altri baroni del Reame e partire al seguito del Vicerè per la guerra di Siena, non senza prima aver fatto un nuovo secondo testamento presso il notaio del luogo Giacomo Manarino.' Il nuovo indizio, insomma, costituito dalla presenza di Galeazzo entro questa raccolta che a Siena fa riferimento, incoraggia l'ipotesi (lungimirante, occorre dire) avanzata dal Bozzetti ${ }^{10}$ che il codice Cavalcanti, posto a base dell'edizione Spiriti del 1758, provenendo dalla biblioteca di San 


\section{MASSIMO DANZI}

Giovanni in Carbonara, dove erano finite le carte della famiglia Martirano, potesse essere stato donato dall'autore "in segno di omaggio, o gratitudine e stima" a Coriolano Martirano, controfirmatario dell'atto di grazia con il Vicerè Pietro di Toledo, e costituisce al contempo anche l'unica, preziosa testimonianza della penetrazione della sua poesia in terra di Siena.

Da questo punto di vista, nuovi elementi aggiungono le rime dell'accademico "Deserto", fra le quali alla carta 27 recto si ritrova adespoto il testo di Galeazzo. Il sonetto di Galeazzo è infatti immediatamente seguito nel codice da uno analogo dell'accademico Intronato, che appare essere una bella e stretta imitazione del modello, pur piegata alle esigenze di una destinataria con pastorale nome di "Flori", della quale si verifica facilmente la presenza fra le rime del Barozzi. ${ }^{11}$

Pubblico, nell'ordine, la redazione del testo di Galeazzo testimoniataci dal codice senese $e$ il sonetto che immediatamente lo segue con esplicita attribuzione "Del Deserto Intronato". L'imitazione che questo secondo attua del modello tarsiano risulta evidente e non necessita, mi pare, di un particolare commento:

Come in limpido vetro, $\mathrm{o}$ in onda pura se 'l destro occhio del Ciel rif[l]ette in lui mirar si può, quel che nei raggi sui debil vista mirar non s'asicura,

così la mia ch'altro veder non cura perde (ò lume gentil) mirando in vui che quasi un nuovo sol sete fra nui, ch'occhio non sano à gran splendor no(n) dura;

ma s'io mi volgo al cor che d'ogni parte riceve la di voi luce in se stesso, non è sì chiaro in lei ch'ei non si mostri;

dunque a che tormi il sol de gli occhi vostri

s'io lo veggio assai men s'a lui m’appresso e lo scopro da lunge a parte a parte?

Ed ecco, invece, il Barozzi:

Come pur[o] cristallo in cui del Sole chiaro splendor $\mathrm{p}(\mathrm{el})$ Ciel seren discende, mentre l'avuta luce indietro rende ciò che tocca con quella infiammar suole, 
così di quello eterno et vero sole

da cui el lume el girar quest'altro prende,

sì vivo raggio ne' begli occhi splende

di Flori, che mi sface et non men duole;

che poi, volgendo in voi di quei la luce,

occhi mortal non è che la sostegna

ogni cosa infiammando, o lungi o appresso.

Io, che viver non posso ove non luce

quella, d'avanti a lei quel ch'io divenga

lo dica Ella et Amor che 'l veggio spesso.

Il testo di Galeazzo che il manoscritto senese (d'ora in poi S) ci dà, presenta, con evidenza nelle terzine, un'altra redazione del sonetto: la qualità delle varianti, che in una buona metà dei casi lo oppongono a tutta la tradizione, non permette di dubitarne. Al v. 2 e al v. $6, S$ ha rispettivamente "rifll]ette» e "(o lume gentil)" dove gli altri testimoni concordemente leggevano "risplende» e "Donna real». Al v. 7, nuovamente isolato rispetto alla tradizione che aveva "che siete un nuovo sole oggi fra nui», S reca "che quasi un nuovo sol sete fra nui». Notevoli, e certo "redazionali", anche le varianti delle terzine. Nella prima, ai vv. 10-11, $S$ si stacca da una varia lectio che, senza infrangere tuttavia la compattezza della tradizione, leggeva: "riceve la di voi luce in se stesso I non è si chiaro in lei chei non si mostri); nella seconda, al v. $13 \mathrm{~S}$ legge "s'io lo veggio assai men s'a lui m'appresso", lezione che rappresenta un esito intermedio fra le soluzioni di $C$ da una parte (use il veggio meno assai qualor l'affiso") e la lezione data a testo, che è (con lievi oscillazioni formali) dei restanti testimoni ("se il veggio assai via men se in lui m'affiso"). Infine, al v. 14, di nuovo $S$ si oppone a tutta la tradizione sostituendo "da lunge» alla lezione «in me stesso»: "e lo scopro da lunge a parte a parte».

Fin qui, senza bisogno di considerare le varianti formali, quanto appartiene alla redazione di S. Ma il codice di Siena, se percorso interamente, rivela uniulteriore sorpresa, che interessa ancora congiuntamente la storia del poeta calabrese e del suo imitatore toscano. Alla c. 38r, infatti, quella che a me pare un'altra mano copia, con ductus più trasandato e frettoloso, due frammenti apparentemente privi di forma, ma tuttavia riconducibili con evidenza ai nostri due testi.

Prima di trascrivere il contenuto di questa carta esattamente come si presenta (si veda la riproduzione fotografica), con l'apparato delle cor- 
rezioni o inserzioni del copista, ricordo che la stessa carta risulta a tutti gli effetti aggiunta, come dice oltre che il formato diverso anche la filigrana, che è unica in tutto il manoscritto e rappresenta qualcosa di assai simile alle corna di un bue, fra le quali sinnesta una stele con in cima una stella a quattro punte. Tale filigrana non è documentata nella raccolta del Briquet. Ma ecco il contenuto della carta:

Come $i(n)$ limpido vetro o in onda chiara

sel bel lume del sol riflette i(n) lui

si scorge in parte quel vago splendore

ch'in se stesso mirar alcu(n) non puote

così quel bel ch'in voi natura pose

vo rimirando nel mio cuor sove(n)te

ch'ivi Amor la scolpì co(n) la sua mano

e riceve da voi ogni suo lume

ma s’io riguardo in voi tanta è la luce

de sereni occhi vostri che mirarvi

io pur no(n) posso e questo è certo

ch'occhio no(n) san à gra(n) splendor no(n) dura

quando ti son apresso ogni mio senso

intento è à procurar se in te propria

potesse mai conoscere quel bello

ch(e) v'ascose natura o(n)de tu fusse

esempio al'altre oltre à le belle bella

onde nel cuor io non ti miro al'hora

né l'occhio può mirarti e resto privo

di così bel vedere onde se noia

t'è ch'io ti veggia stammi ogni hora à presso

ch'el mio senso visivo no(n) vedratti

e men nel cuor potrò mirarti mai

3 quel con q- da il - 8 tutto il verso pare aggiunto in interl. - 9 tanta con -a da -e - 18 io con i da $\mathrm{p}-20$ nel $m s$.

Il secondo frammento, che solo per ragioni di spazio il copista dispone su due colonne, legge: ${ }^{12}$

Come puro cristallo

in cui scende dal sole

una serena luce io son nuova fenice

ch(e) nasce e vive i(n) fuoco

e tanto è la sua vita 
mentre l'hauto lume

5 lucido indietro rende ciò ch(e) tocca co(n) quello inffiamma arde e abrucia così sono i miei occhi $\operatorname{ch}(\mathrm{e}) \mathrm{da}$ le vostre luci

10 prendon qua(n)to han di luxe infiamman e foco fanno il mio $\operatorname{sco}(\mathrm{n})$ tento core riscaldato da quelle luci $\operatorname{ch}(\mathrm{e})$ in lui riflette qua(n)to la fiamma dura

finito il fuoco et ancor lui si muore

et io n'acqui nel fuoco

del' amoroso telo

ch(e) vivo no(n) fui mai

mentre fui fuor del vostro arde(n)te fuoco qua(n)to sto apresso à voi tanto son vivo se mi state da lungne

7 arde (o anche ?) aggiunto in interl. -8 abrucia con -c- su -s- -9 luci con -i su -e 10 luxe da luxi [ma 10-11 potrebbe anche leggersi: luci e / in fiamma e foco fanno] - 11 e foco fanno sostitutivo in interl. di e in fuoco volge cass. -25 lungne da lunge -26 forza ch' io con i su e

Come dice anche il rigo di copista che taglia orizzontalmente la pagina in due parti, i due frammenti vanno considerati separatamente. Il primo, 23 versi (tutti, tranne il più incerto v. 11, endecasillabi), contiene gli elementi, nonché le principali tournures sintattiche del sonetto di Galeazzo. Il secondo, a prevalenza di versi settenari con tre soli endecasillabi ai vv. 19, 24-25, rinvia invece con evidenza al sonetto del Barozzi, che segue e imita quello di Galeazzo.

La numerazione che do del frammento riportabile a Galeazzo intende evidenziare gli snodi sintattici comuni fra sonetto e questi versi, con un rapido espediente. Ma se è evidente la comunanza di molti elementi, assai piu problematico risulta il riconoscimento della direzione in cui procede il lavoro di chi ha trascritto questo manipolo informe di endecasillabi. Presento, per il frammento relativo a Galeazzo, quelle che mi sembrano le ipotesi plausibili, affrontando in un secondo momento la questione di quello del Barozzi.

Due mi sembrano essere, sostanzialmente, le possibilità di interpretare questo materiale e fra queste due ipotesi sinserisce il dubbio che, così come questi ventitrè endecasillabi si presentano, essi siano non già una semplice trascrizione (eviterei fin d'ora l'ipotesi della autografia), bensì il risultato di un accorpamento di frammenti, questi sì forse autografi di Galeazzo, operato dal copista che li raccolse trascrivendoli di seguito. Questa terza possibilita, vedrebbe insomma un copista riunire dei tentativi che l'autore 
stesso aveva probabilmente annotato e che si presentavano non già come un unico testo, bensì come tentativi reiterati di singoli spunti testuali. Da qui, probabilmente, deriverebbero le ripetizioni $e$ incongruenze che nel frammento sono evidenti.

Questo dubbio sostanzialmente fa corpo con la prima delle due ipotesi, secondo la quale il frammento potrebbe essere a tutti gli effetti una redazione primitiva, ancora informe, del sonetto che conosciamo. Insomma, da un primo getto (autografo, o più probabilmente no) ancora informe se pur già sostanzialmente calibrato sull'endecasillabo si passerebbe alla soluzione formalmente definitiva del sonetto, allo stesso modo in cui da un blocco ancora grezzo uno scultore perviene a trarre il volume che gli interessa. In questa ipotesi Galeazzo penserebbe e opererebbe fin dall'inizio in versi, ciò che corrisponde assai bene a quanto del lavoro, per esempio dell' Ariosto, è stato esemplarmente illustrato da Contini. ${ }^{13}$ Se questo è l'iter, il passaggio alla forma finale del sonetto può avvenire invertendo anche la successione degli elementi, come dimostra il confronto fra i vv. 9 e 12 del frammento, (d'ora in poi A: "ma sio riguardo in voi tanta è la luce / (...) I ch'occhio non san a gran splendor non dura") con la progressione del sonetto, nella redazione del manoscritto senese: "chiocchio non sano a gran splendor non dura I Ma s'io mi volgo al cor che d'ogni parte / riceve la di voi luce in se stesso" (vv. 8-10). Certo, pare di vedere un acquisto di indeterminatezza nella rappresentazione, con l'abbandono di un lessico più "fisico" del frammento. ${ }^{14}$ Solo "riflette», $v .2$ di A, resiste ancora in $S$ per poi essere sostituito in NS con "risplende»; le altre sostituzioni simpongono tutte nel passaggio da $A$ a $S$ ("onda chiara», v. 1, diviene "onda pura», v. 1 ; «si scorge», v. 3, diviene "mirar» entro un distico (vv. 3-4) toccato parallelamente dalla cancellazione di un non poi troppo indeterminato "alcuno" e dalla sostituzione in perifrasi dei vv. 3-4 del frammento: da usi scorge in parte quel vago splendore / che in se stesso mirar alcun non puote» di A si passerebbe a "Mirar si può quel che ne raggi sui / debil vista mirar non m'assicura" di $S$ e poi NS. Il risultato è l'insistenza di Galeazzo su "mirar» ("mirar si può»v. 3, "mirar non s'assicura»v. 4, "mirando in vui» v. 6), verbo chiave in un sonetto di contemplazione dell'amata com'è questo, espressione - mi pare - di un Galeazzo fortemente concettuale e manierato. Emerge il senso principe della vista e le quartine guadagnano una più ossessiva e immobile rappresentazione della donna, rispetto alla difratta e temporanea "fisicità" del presunto abbozzo ("si scorge» v. 3; "mirar alcun non puote» v. 4; "vo rimirando" v. 6; "ma s'io riguardo" v. 9, ecc.).

Sto ragionando, lo ricordo ancora, come se $A$ (pur nello stato in cui si 
presenta) fosse l'abbozzo del futuro sonetto di Galeazzo, abbozzo raccolto da qualcuno che può anche averne resa unitaria una fisionomia non tale all'origine. Tale ipotesi, che se ricostruita entro $A$ imporrebbe il frazionamento del frammento in segmenti successivi il cui discrimine potrebbe ipotizzarsi nella replicazione dei concetti entro le stesse tournures (bastino i vv. 5 e 16 col motivo di Natura che dispensa ogni bellezza nella donna), ${ }^{15}$ obbliga ad osservare che vario è il materiale del frammento sacrificato nel sonetto, ${ }^{16}$ che nella redazione più antica di S ricupera al $v$. 8, cioè a chiusa delle quartine, l'originario verso 12 di A: "ch'occhio non sano a gran splendor non dura", cioè l'unico verso memorabile per sapore sentenzioso e che dunque passa ne varietur la trafila elaborativa. Il recupero fissa definitivamente fronte e sirma del sonetto e forse impone la catena rimica in -ura, attiva nella sostituzione di "onda chiara" con "onda pura" dell'incipit, con un sintagma cioè che, per essere un unicum di Galeazzo, collega il sonetto alla canzone A qual pietra somiglia (Rime XIX), "lapidario amoroso" nel quale il Bozzetti ha ravvisato proprio il vertice del "virtuosismo tecnico raggiunto da Galeazzo nella poesia della lode». ${ }^{17}$ In quest'ottica, cioè nell'ipotesi che A preceda e confusamente accumuli, magari accorpandoli indebitamente, i futuri materiali di $S$, il passaggio del v. 12 in ottava sede nel sonetto fa corrispondere naturaliter quelli che erano i versi 9-11 del frammento ("ma s'io riguardo in voi tanta è la luce, I (...)») alla prima terzina del sonetto ("Ma s'io mi volgo al cor che d'ogni parte (...)川), che in questo luogo capitalizza forse anche la suggestione dei vv. 19-21 del frammento. E, occorre osservare che, rispetto alla lezione raggiunta in $S$, l'autore opererà a questo punto solo minimi ritocchi nel lessico e nella dispositio, a significare che ormai il lavoro di vera e propria inventio $\dot{e}$, all'altezza della redazione senese, tutto o quasi risolto. ${ }^{18}$ L'abbandono, che pare ravvisabile, del tono più fisico e soggettivo di $A$ a favore di una maggiore oggettività della rappresentazione, attenua simpateticamente anche la presenza dell' io del poeta, vivace nel frammento ("vo rimirando» v. 6, "ma s'io riguardo" v. 9, "io pur non posso» v. 11, "io non ti miro" v. 19, "se noia t'è ch' io ti veggia" vv. 21-22, ecc.), dove per altro la presenza della donna non andava oltre la materialità del pronome, oscillando per di più fra un "voillei" della prima parte e un "tu» della seconda. É questa un'importante caratteristica di $A$, che difficilmente può attibuirsi ad una redazione conclusa del testo e che invece accrediterebbe piuttosto l'ipotesi di A come "abbozzo" dalla fisionomia non unitaria. ${ }^{19}$ Diverso il comportamento di $S$, e poi anche più di NS, che alla donna si rivolgono con il "voi»: ivi, la donna guadagna in concretezza e da un vocativo ancora catafratto e generico "(o lume gentil)" v. 6, giunge, 
in NS (e nell'intera tradizione), al più concreto ed esplicito "Donna real", con un acquisto che agisce orizzontalmente collegando il sonetto ad almeno quattro altri testi della raccolta, fra $i$ quali uno, il XXXVII, originariamente composto per Giovanna d'Aragona. ${ }^{20}$ L'elemento è minimo, ma mi pare possa inserirsi fra quelli che definiscono modalita precise della costruzione a "canzoniere", che Galeazzo otterrebbe attraverso l'estensione di tessere comuni finalizzate all'omogeneizzazione del linguaggio della raccolta. Infine, è acquisto definitivo di $S$ (o, se si preferisce, è già in S) anche l'interrogativa diretta che chiude il sonetto. Essa per un verso risolve, entro il giro di una terzina, i modi piu incerti e cincischiati di A, vv. 19-23 ("onde...onde», ecc.), per altro verso instaura un ulteriore preciso collegamento con due sonetti (XXXIII e XXXVI), che soli nel canzoniere adottano quel tipo di chiusa.

Prima di presentare la seconda ipotesi in ordine al frammento, vorrei tuttavia richiamare i problemi che sorgono nel giudicare la natura del frammento $A$.

Di Galeazzo di Tarsia, come di molti anche eccellenti autori del Cinquecento, non conosciamo scritti autografi; e non pare che la sua grafia ci sia serbata neppure dai testamenti che di lui sono noti. ${ }^{21}$ Se, come detto, rari, in questo secolo, sono gli autografi poetici che conserviamo, anche piu rari sono $i$ casi in cui materiali di un poeta si presentino con le caratteristiche dello "scartafaccio", mettendo in condizione lo studioso di penetrare nel laboratorio di un autore. Da questo punto di vista, per quello che è noto oggi, il caso celebre dell'Ariosto e quello altrettanto famoso, ma meno studiato, di Michelangelo, costituiscono nel primo Cinquecento due luminose, ma relativamente isolate, eccezioni. Di recente, ad essi si è aggiunto il caso cospicuo del Tebaldeo, studiato nella loro edizione dalla Basile e dal Marchand. ${ }^{22}$ L'ipotesi che a questa breve lista possa aggiungersi il nome di Galeazzo, non mi pare probabile per quanto riguarda il piano dell'autografia di questo frammento. Mi sembrerebbe invece per lo meno possibile per quanto attiene alla natura del frammento stesso, quale ho cercato di caratterizzare. $M a$, se il frammento non è autografo (come mi parrebbe certo), resta da spiegare chi, raccogliendo probabili tentativi autografi del poeta cosentino, lo abbia riunito o anche solo trascritto. Ed è risposta che non so dare.

Si presenta, a questo punto, quella che può essere una diversa spiegazione della natura di A. Per brevemente illustrarla, devo ricordare che la carta aggiunta nel codice che ospita il frammento anche ospita, nella sua parte inferiore, la redazione diversa, che ho sopra trascritto, di un testo sicuramente riconducibile all'accademico intronato Antonio Barozzi. Sui 
rapporti che intercorrono fra frammento e sonetto, vale, mi sembra, quanto ho detto per Galeazzo. A ben guardare, anche il frammento del Barozzi non dà un testo finito e nemmeno, forse, unitario stando alle ripetizioni e incongruenze non lievi che lo caratterizzano. A rigore, dunque, se per Galeazzo è possibile pensare ad un abbozzo, potrebbe essere questo il caso anche per il testo del Barozzi. Ma, se invece pensiamo che la progressione per il Barozzi sia altra, e cioè proceda dal sonetto Come puro cristallo in cui del Sole verso il frammento più informe (e libero da rime) che segue quello tarsiano, allora anche per Galeazzo non può evitarsi l'ipotesi che non di abbozzo si tratti, bensì di un rifacimento. Rifacimento, o riscrittura, per entrambi gli autori in versi sciolti, autore (forse) lo stesso Barozzi. In questa seconda ipotesi, punto di partenza è ancora il sonetto di Galeazzo che il Barozzi prima imiterebbe con l'analogo suo e del quale poi tenterebbe una riscrittura di getto e con qualche incidente e incongruenza evidente. Parallelamente, Barozzi attuerebbe la riscrittura in sciolti anche del suo sonetto-imitazione, sulla stessa carta (27r della nuova numerazione), avendo cura di separare con una linea orizzontale $i$ due rifacimenti, originati entrambi dall'imitazione di Galeazzo e dunque confondibili. Se così fosse, i materiali che il codice di Siena ci consegna non sarebbero abbozzi ancora informi di due futuri sonetti, bensì esperimenti di riscrittura in sciolti a partire dal testo del Tarsia e, dunque, per ciò che riguarda Galeazzo, testimonierebbero non del laboratorio del poeta cosentino, ma della fortuna senese di un suo testo. ${ }^{23}$

Nell'una e nell'altra ipotesi, la mano che copia questa carta aggiunta è quella di un copista colto, come dice sul recto della carta la trascrizione, ad opera sua, di un brano di commento in latino al De generatione et corruptione di Aristotele, ${ }^{24}$ di un autore cioè, Aristotele, che insieme ad altri classici può ben appartenere alla cultura del Barozzi, quale appare dalle sue Lezioni accademiche, leggibili nel codice H.IX.37 della stessa biblioteca senese.

La prigionia di Galeazzo a Lipari prima, la sua partecipazione alla guerra di Siena poi, anticipano il senso di una vita che sappiamo tragicamente conclusa, per mano omicida, a trentatrè anni, nel 1553. Tali vicende biografiche non devono aver mancato di riflettersi anche sulla tradizione delle sue rime, determinandone (con altri fattori a noi oggi ignoti) una cosi scarsa circolazione. Che di lui Siena restituisca ora la testimonianza piu arcaica di un sonetto insieme a un piu problematico frammento in sciolti, è fatto che, al di là dell'interpretazione che se ne può dare (abbozzo o "riscrittura"), la pietas dello storico registra.

Massimo Danzi 


\section{Massimo DANZI}

1. Galeazzo di Tarsia, Rime, edizione critica a cura di C. Bozzetti, Milano, 1980. Tre i manoscritti e due sole le stampe alla base della ricostruzione del Bozzetti, secondo cui del canzoniere tarsiano ci «sono pervenute due forme diverse: una più antica, rappresentata $\mathrm{da} V \mathrm{e} d a$ NR in due fasi successive della sua strutturazione; una più tarda e profondamente rimaneggiata e arricchita, rappresentata da NS; in più $\mathrm{C}$ ci conserva probabilmente un residuo del lavoro preparatorio almeno per le fasi V e NR» (GAleazzo di TARSIA, Rime cit., p. XXVII). Per le sigle vedi la nota seguente. Su Galeazzo importante è lo studio biografico di Carlo De Frede, Il poeta G. di Tarsia signore feudale di Belmonte, "Archivio storico per le provincie napoletane» s. III, 2, 1962, pp. 7-107.

2. Le sigle rinviano ai seguenti testimoni: $\mathrm{C}=$ Chicago, Newberry Library, Case ms. 6A $11 ; \mathrm{V}=$ Roma, Biblioteca Apostolica Vaticana, Reginense Lat. 1603; NR = Rime di Galeazzo di Tarsia Nobile Cosentino. Raccolte Dal Cavalier Basile Nell'Accademia de gli Otiosi il Pigro, In Napoli, 1617, Per Gio. Domenico Roncagliolo; NS = Le Rime di Galeazzo di Tarsia Cosentino Signor di Belmonte In questa nuova Edizione accresciute e ridotte alla loro vera lezione, col ritrovamento di un antichissimo M.S. e con la Giunta di alcune Osservazioni, e della Vita dell'Autore, In Napoli MDCCLVIII, Nella Stamperia Simoniana. Aggiungo che, nel caso dei due codici che qui interessano per il sonetto XVI, cioè $\mathrm{C} \mathrm{e} \mathrm{V}$, la mano del copista (almeno per la parte che interessa Galeazzo) è giudicata dal GAlEAzZO DI TARSIA, Rime cit., p. XX, rispettivamente "tardocinquecentesca" e "della seconda metà del ' 500 ".

\section{Galeazzo di Tarsia, Rime cit., p. 53.}

4. Galeazzo di TARSia, Il Canzoniere. Nuova edizione corretta su tutte le stampe con note ed uno studio sull'autore di F. Bartelli, Cosenza, 1888, a p. 41 delle Annotazioni al canzoniere. La parafrasi del Bartelli è ripresa nei Lirici del Cinquecento, a cura di D. Ponchiroli. Nuova edizione a cura di G. Davico Bonino, Torino, 1968², p. 548, e in quelli a cura di L. Baldacci, Milano, 1975², p. 497.

5. Galeazzo di Tarsia, Rime cit., p. 53.

6. L. Ilari, Indice per materie della biblioteca comunale di Siena, Siena, 1845, vol. I, p. 184 e P. O. Kristeller, Iter italicum, London-Leiden, 1966, vol. II, p. 168.

7. Si tratta di un codice miscellaneo, composito, di diverse mani, di mm. $292 \times 207$, costituito da cc. II + 59 + IV (la I e la IV riutilizzano pergamene liturgiche due-trecentesche). Tutte le carte, tranne l'attuale c. 38, recano in filigrana un giglio, identificabile con quello di Briquet, n. 6897 [1555]. Altra invece (e non reperibile nel Briquet) la filigrana della carta 38, importante per Galeazzo, che risulta anche per il formato (mm. 276 x 200) aggiunta. Sulla costola di copertina reca l'intitolazione "Poesie volgari latine sec. XVI". L'indice di mano moderna (Ilari ?), a c. II r, registra solo i nomi esplicitamente presenti nel codice (Ludovico Ariosto, Annibal Caro, Claudio Tolomei, Lattanzio Tolomei, Francesco Maria Molza, Niccolò Scevola, Madonna Chiara Gentildonna Lucchese [ciò̀ la Matraini], Giovan Bernardo Rosci, Giovan Francesco Spannocchi, Il Deserto intronato [ciò̀ Antonio Barozzi], Il Cieco intronato [cioè Camillo Falconetti], Latino Latini, Francesco Piccolomini, Mons. Capello, Virginia Salvi, Mons. Della Casa, Tullia D’Aragona, Giovanni Rosa, Luigi Alamanni, Vittoria Colonna, Giacomo Grifolo). Usa e descrive brevemente il codice Giovanna Rabitti nella sua edizione critica di ChIARA Matraini, Rime e lettere, Bologna, 1989, pp. LXXXVII-LXXXIX (con bibliografia pregressa).

8. Qualche esempio, avvertito che ragiono naturalmente sulla tavola completa del manoscritto: c. 1r «1540», 13r «Di Vigevano al'ultimo di Marzo nel 1546»; 23v «dal Poggio, il MDL il XXV di gennaio"; $24 \mathrm{r}$ "Son. in morte del Rmo Cardenal Bembo [18 gennaio 1547 n.d.r.] da Napoli venuto»; $26 \mathrm{r}$ "da Milan son venute tutte queste [sc. composizioni] del cagniolino di Don 
Hippolita [Gonzaga n. d. r.]»; 27r "da Fiorenza venuto»; 28r "Da Roma m. Latino Latini»; 30v "1552 di Dicembre»; 31v "Di gennaio 1552"; 32r "Da Roma non si sa l'autore"; 34r "Genn. 1553 Epigrammi di L. Alamanni di Francia venuti» Genn. 1553; 41v «Di Napoli venuto»; 59v «In linguam maledicam. Rome actum» ecc.

9. Sui crimini e la condanna a Lipari di Galeazzo, cfr. F. Bartelli, Note biografiche (Bernardino Telesio - Galeazzo di Tarsia), Cosenza, 1905, pp. 168-69. La notizia della partenza per la guerra di Siena, poi accettata dagli studiosi fino a L. Baldacci, Lirici del Cinquecento, Milano, 1975², p. 490 e a Galeazzo di Tarsia, Rime cit., p. XXV, sempre in Bartelli, Note cit., pp. 174-85. Sul testamento di Belmonte, che rende erede universale la figlia Juliella, cfr. ibid., pp. 178-79. Secondo i documenti studiati dal Bartelli, Galeazzo "non morì sul campo, come sostenne qualcuno (...), ma poco tempo dopo il suo ritorno dalla Toscana, a primi di giugno [del 1553]", cfr. ibid., pp. 184-85.

\section{Cfr. Galeazzo di Tarsia, Rime cit., pp. XXIV-XXV.}

11. Su di lui, cfr. Gianmaria Mazzuchelli, Gli scrittori d'Italia, Brescia, presso G.B. Bossini 17531763, vol. II, pp. 409-10. Leggo le rime del "Deserto" in tre manoscritti principali, tutti del sec. XVI (Ferrara, Bibl. Ariostea, N.A. 5; Firenze, Bibl. Nazionale Centrale, Palat. 256 e il più importante Magl. VI 1389) e anche nel Casanatense 897, mentre le stanze sono poi a stampa nell'importante, per gli autori senesi, Primo volume della Scielta di Stanze di diversi autori Toscani raccolte da $M$. Agostino Ferentilli et di nuovo con ogni diligenza ricorrette, in Venetia, appresso Filippo e Bernardo Giunti \& fratelli, MDLXXIX, pp. 34-44. Importa per la conoscenza dell'opera del Barozzi anche il ms. H.IX.37 della Biblioteca Comunale di Siena, che contiene le sue Lezioni accademiche discorsi di tema "amoroso" costruiti come commento a passi soprattutto del Petrarca volgare (e la mano di questo codice - autografo? - pare assai vicina a quella che copia i sonetti di Galeazzo e del Barozzi), mentre i Dieci Paradosse degli Accademici intronati da Siena, In Milano, Appresso Gio. Antonio degli Antonij, 1564, si aprono con un dialogo accademico di argomento amoroso fra lui e "lo Scacciato», cioè Marco Antonio Cinuzzi (cc. A 3r-B 1r). Non merita citare qui la ricca ma diseguale bibliografia sull' Accademia.

12. In corsivo i luoghi del testo per i quali la lettura non è sicura.

13. G. Contini, Come lavorava l'Ariosto, in Esercizî di lettura sopra autori contemporanei con uniappendice su testi non contemporanei. Nuova edizione aumentata di Un anno di letteratura, Torino, 1974, pp. 232-41. Alla p. 235, la celebre rivendicazione della "contemporaneità, e non anteriorità, dell'ispirazione rispetto al metro".

14. Chiamo $\mathrm{A}$ il presunto primo getto rappresentato dal frammento, $\mathrm{S}$ la redazione del sonetto testimoniataci dal codice senese e NS il sonetto nella redazione a testo dell'edizione Bozzetti.

15. Ecco i vv. 5 e 15-16 di A: "così quel bel ch'in voi Natura pose» e "quel bello / che v'ascose Natura". Ma il carattere non unitario di A è ipotizzabile anche per altri elementi: certe ripetizioni che parrebbero "alternative" ("onde ... onde» vv. 18-20), certe rotture sintattiche come ai vv. 7-8 e soprattutto l'oscillare (solo del frammento) nel rivolgersi alla donna fra il «voi/lei» della prima parte e il "tu» della seconda. Ma per questo vedi la nota 19.

16. Così, per es., tre versi, indubbiamente riusciti, come "così quel bel ch" in voi Natura pose / vo rimirando nel mio cuor sovente / ch'ivi Amor la scolpì con la sua mano» (vv. 5-7).

17. Galeazzo di TARsia, Rime cit., p. 62. La ripresa del sintagma in questa canzone, v. 6 , reca con sé la rima (facile) onda pura : dura e di lì a poco si riflette in puro intelletto (XXIX 13) e pura / Fiamma 


\section{MASSIMO DANZI}

(L 1-2). Altro unicum del canzoniere («virtù visiva» XIX 11) era in precedenza vivo in A 22: "senso visivo".

18. Si osservi d'altra parte, al v. 10, la prossimità della lezione di S («riceve la di voi luce in se stesso») a quella di C, V e NR: «riceve il folgorar [C balenar] del vostro viso», dove NS avrà, in ordine alla maggiore oggettività che lo distingue, "vago viso».

19. Questa l'oscillazione del pronome in A: «in voi» v. 5, "Amor la scolpì v. 7, «riceve da voi» v. 8 , «in voi» v. 9, «occhi vostri» v. 10 , «quando ti son apresso» v. 13 , «in te» v. 14 , «onde tu fusse» v. 16, "ti miro" v. 18 , «mirarti» v. 19 , «se noia t'è ch'io ti veggia» vv. 20-21, «vedratti» v. 22 , "mirarti» v. 23 , ecc.

20. Questa la rete istituita dal ricorrere dell'aggettivo: «Real sembiante»XIV 14, "real petto gentile» XXXVI 12, «Donna reale» XXXVII 1, «real donne» XXXVIII 2.

21. Il primo del 1551, notaio Napoli della Macchia, Archivio notarile di Cosenza; il secondo del 24 settembre 1552, notaio Giacomo Manarino, Archivio di Stato di Napoli (su entrambi, da ultimo, Bartelli, Note cit., pp. 109-10). Ripubblicando il testamento del 1552 alle pp. CVIII-CXI del suo studio del 1888, (cfr. n. 4), il Bartelli accenna, per contro, a una procura autografa con la quale, in occasione di un viaggio a Napoli, il poeta lasciò con pieni poteri lo zio Francesco nel castello di Belmonte: «Il prezioso autografo - afferma il Bartelli - è ben conservato e porta sul dorso le parole Procura di carattere di Galeazzo di Tarsian (p. XCIII). Ignoro la base di questa affermazione, che se corrispondesse a verità produrrebbe l'unico autografo. Nulla, in proposito, segnala lo studio del De Frede menzionato alla nota 1.

22. Antonio Tebaldeo, Rime, a cura di T. Basile e J.-J. Marchand, Modena,1989-1992, 5 voll.; vol. I, pp. 145-230. Fra i poeti di primo Cinquecento, dei quali abbiamo stesure autografe ricordo tuttavia almeno i casi del Bembo, del Bandello, del Machiavelli. Ma già del Casa, ad esempio, restano pochissimi versi autografi, accanto al noto apografo rivisto dall'autore che è oggi il ms. Magliabechiano VII.794 della Biblioteca Nazionale Centrale di Firenze.

23. Osservo tuttavia che, se l'uso del verso sciolto a metà del Cinquecento non pone problema, non ne abbiamo invece testimonianza nell'opera, manoscritta e a stampa, del Barozzi. Sotto il profilo linguistico, d'altra parte, nei due frammenti all'assenza di forme meridionali si contrappone almeno un forte toscanismo come «hauto" per havuto, al v. 4 del secondo frammento.

24. Inc: "[de i] llis que ad omnia naturalia consequuntur sic in libris de generatione et corruptione pertractat, ecc.». 


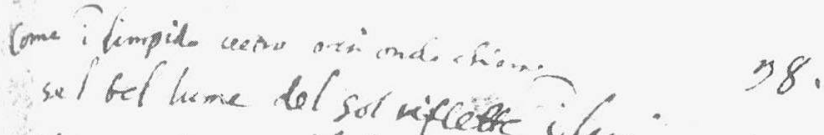

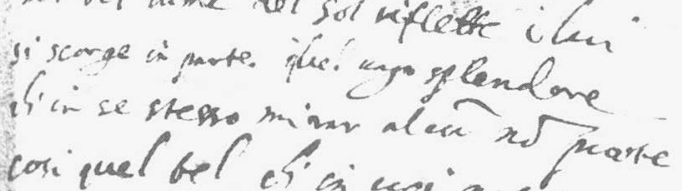

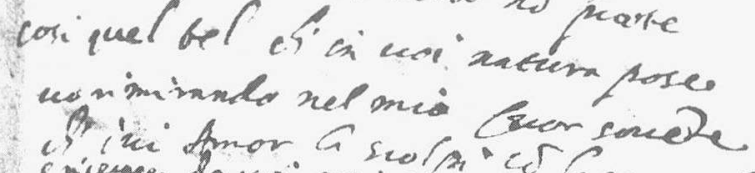

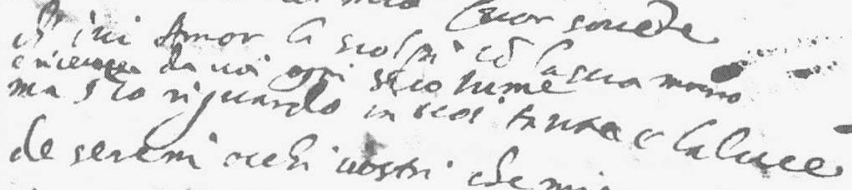

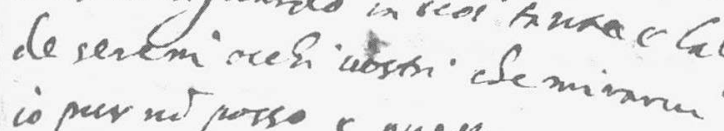

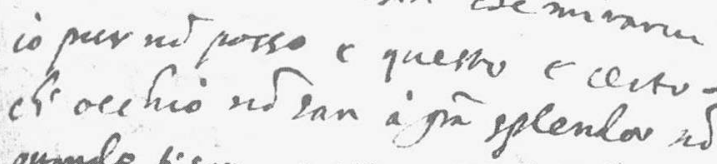

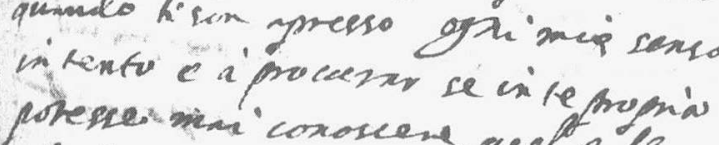
poremerna conoscene nuel fello th incuse naturn Jle. F fiesso crempia al athe olore i ce belle fell.

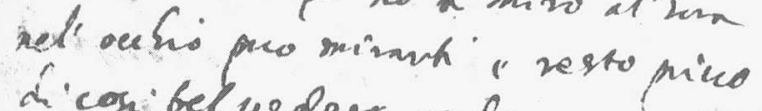
dicosibelwedere oxde of xera.

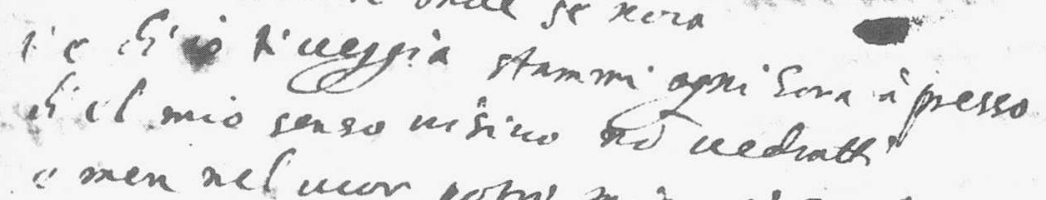

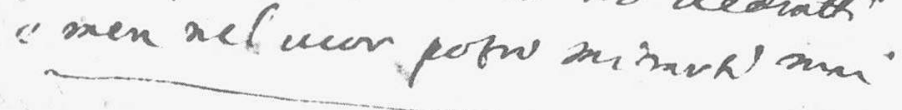

tane pan on! trllo

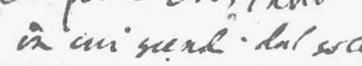
ime serese lace nente lisustoluma lundo nibieno nole: lio Iroceref puatlo.

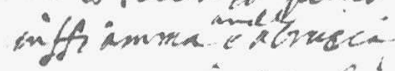

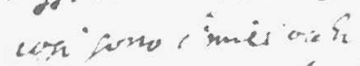
it ha le nothe hies.

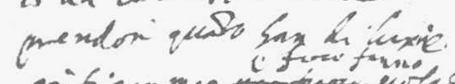

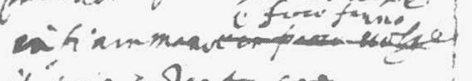
$y$ "ing icstento gom

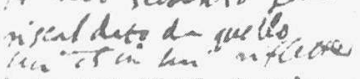

ic som man pincio

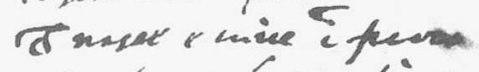

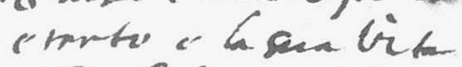
vioto la fesoundara.

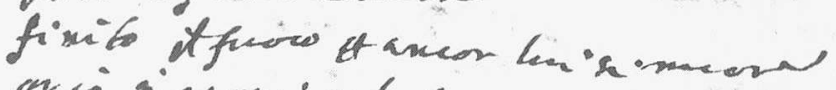

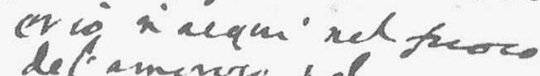
detameno nel is mino is far ma.

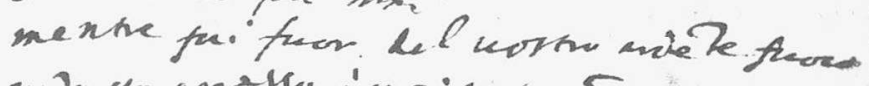
quato to apresth a'uer thato is mices re mistate th langeres.

4

4 
Sont in limpide uetro on onda guna

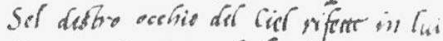
- 11 urar si quo, quel che nei roggi sui obbil wha mirar non s'asioura

Cosi la mia shi abro wedir non ewra.

Iseder à Gomr genrif mirandw in un

Cho quasi un mious sol sere frot miv ch occhi non sano à gron splerdor at wo cura

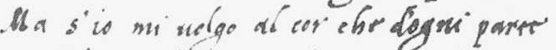

Riverir la pi noi hut in se kesso.

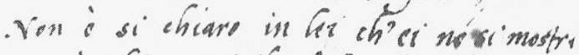

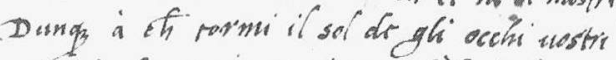

" $s$ is is ugaw assai muat s'alus mispresso

$r$ io soper ofa cunge esparpe a parte.

$$
\text { dof Deservo Terrosiato }
$$

Cemi porro cristallo in usi del sole

Chare splender a ciel seren disecnde it creve fi haunta bue sndictro renis

- Ciechir rocea cosi quila infiammar such cosi di quello ereme \&- usio sole

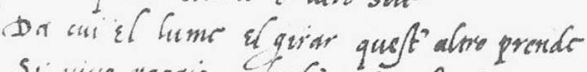

Si wise raggio ne vegti occhi splendi

Di fione chr mi sfuer en non men duofo

Che per woifsnde in wer di quer la cuee

occhio morrar non i. shr la sofigna

Ogni cosa infiammando, tungi, nppresso

- che muer non posso our non hat

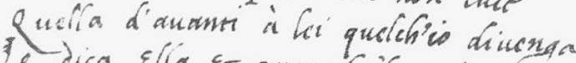

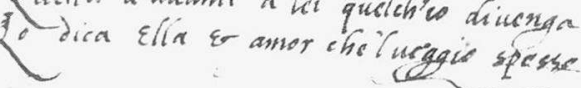

Do spenue, da belor, da wiun froe

Mossa colit Al eui bel nome bonete

Rende haggi if monde, urerne al suo Scancre

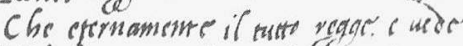

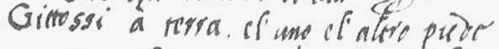

Humitment lanis col calde Gumore

Dorgli ocelit suoi obse di laseine Ansore

Tur prima efea, lacciudi albergo o piedr.

Soi con le sparse eh iemo rascusgolfi.

t uimea dal diaino. Anos'e interno

Con ato rimoente épis baciolli

Felise donna it eui hamil seruire

Tanre piacque al Sianor che premio ererse

Hefore non ef perbon def sue frefire.

CDa fiorenza ucnum

A Mons, d'Anghiono, Goncrate del Re di, fram,

Seper airen affl honorates spada

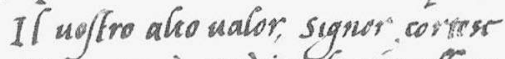

si pronto, à undicar lo wire affeso

con mille pestme at Cus wolond uada Comra a chidiben far, rostaba la strada

vimico ereviso al uno almo pass Mostrate al fine hos áthe usalis acesse

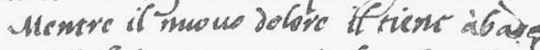

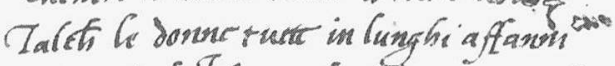

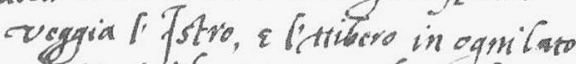

vestine abrume ancor bautarla quanteia

(Casi uoftra merice Jopgo tanti antion

redrem tornare ar sus fiovito stats :

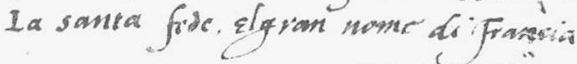

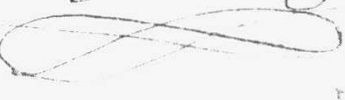

$\because .6 \% 8 \%$ 